SCHNEIDER, Hans, Der fremde Arndt. Studien zu

Leben, Werk und Wirkung Johann Arndts (1555-1621)

\title{
Philippe Büttgen
}

\section{OpenEdition}

\section{Journals}

Édition électronique

URL : http://journals.openedition.org/ifha/535

DOI : 10.4000/ifha.535

ISSN : 2198-8943

Éditeur

IFRA - Institut franco-allemand (sciences historiques et sociales)

Référence électronique

Philippe Büttgen, « SCHNEIDER, Hans, Der fremde Arndt. Studien zu Leben, Werk und Wirkung Johann Arndts (1555-1621) », Revue de l'IFHA [En ligne], Date de recension, mis en ligne le 01 janvier 2007, consulté le 22 septembre 2020. URL : http://journals.openedition.org/ifha/535 ; DOI : https://doi.org/ 10.4000/ifha.535

Ce document a été généré automatiquement le 22 septembre 2020.

(C)IFHA 


\title{
SCHNEIDER, Hans, Der fremde Arndt. Studien zu Leben, Werk und Wirkung Johann Arndts (1555-1621)
}

\author{
Philippe Büttgen
}

1 Parmi les figures hiératiques de la théologie luthérienne à l'orée du XVIIe s., celle de Johann Arndt (1555-1621) continue de luire d'un éclat d'énigme - "Arndt l'étranger » (der fremde Arndt), conclut H.S. au terme de deux décennies de recherche consacrées à la vie et à l'œuvre de l'auteur des Vier Bücher von wahrem Christentum, qui acheva une carrière pastorale mouvementée comme superintendant général à Celle au service du duc de Brunswick-Lunebourg, sans qu'on sache bien aujourd'hui s'il avait reçu une formation théologique suivie. Le nombre de figures marginales, réprouvées, sulfureuses, qui occupent le centre de l'histoire des théologies luthériennes après Luther constitue un phénomène surprenant : on pense aussi à Matthias Flacius Illyricus cinquante ans plus tôt. Les historiens de la théologie ne se distinguant pas par un goût particulier pour les rebelles, il faut penser que cette fréquence du scandale dans l'histoire de ce qu'on appelle, d'une manière qui pourra apparaître de plus en plus curieuse, l'« orthodoxie " luthérienne, est fondée dans l'objet même ; elle a valeur de symptôme de la foncière anomie d'une confession qui, faute de disposer d'instances centrales en fait de politique et de doctrine, restait vouée à une forme de conflictualité permanente au moment même où les dispositions du concile de Trente commençait à être suivi d'effet et où les Églises réformées s'assemblaient en une efficace internationale. Les " Quatre livres sur le christianisme vrai » publiés par Arndt entre 1605 et 1610 constituent l'un des plus spectaculaires best-sellers du luthéranisme : H.S. (p. 135) parle de 200 éditions jusqu'au début du XIXe s., dans à peu près toutes les langues dont le tamoul. Avec leurs contemporaines les Meditationes sacrae (1606/ 1607) du théologien d'Iéna Johann Gerhard, elles constituent le fond de ce que l'on connaît des bibliothèques de pasteurs. Leur interprétation ne les en considère pas moins depuis l'origine comme le réceptacle de toutes les influences que l'orthodoxie entendait rejeter de la droite et saine doctrine : "spiritualisme ", " mystique ", " hermétisme ", à travers la réception de courants comme ceux de la mystique rhénane 
(Tauler), flamande (Thomas a Kempis) aussi bien que d'une philosophie de la nature à vocation médicale (Paracelse, dont le rôle est étudié par H.S., p. 135-155). De ce contraste, il faudra conclure soit que l'interprétation dominante s'égare, soit que les luthériens de l'époque moderne avaient un sens peu assuré de la norme doctrinale. En l'occurrence, les deux conclusions sont sans doute vraies, la seconde valant généralement pour l'exploration de la différence luthérienne dans le système des confessions à l'époque moderne, tandis que la première s'applique plus spécialement à l'évaluation qu'il convient d'opérer du rôle tenu par le Von wahrem Christentum dans une histoire intellectuelle du protestantisme à laquelle sa diffusion confère d'emblée un caractère européen.

Des dénominations comme « mystique » ou " spiritualiste " sont évidemment inaptes à jouer un rôle constitutif dans un travail d'interprétation, sauf quand on les retrouve dans la source, ou non loin d'elle : Zinzendorf, dans une lettre en français au cardinal de Noailles (citée par H.S., p. 82), parle d'« un des Théologiens Mystiques de la Confession d'Augsbourg ". La compréhension du " phénomène Arndt " dans l'univers des théologies confessionnelles du premier XVIIe s. devrait alors reposer sur une sémantique précise des catégories de theologia mystica ou acroamatica au moins depuis Gerson, qui manque encore du côté protestant. À défaut, la solution la plus fréquemment adoptée par les exégètes du Von wahrem Christentum a alors été de souligner son caractère précurseur, piétiste avant la lettre, du fait de l'accent mis dès le premier livre sur la nécessité d'une religion réellement pratiquée, au service d'un renouvellement intérieur de la créature. Il faudra donc admettre que l'un des livres les plus lus au temps de l'orthodoxie luthérienne ait été en fait déjà piétiste : solution inconfortable qui, pour sa part manifestement absurde, renvoie aux limites de la périodisation, toujours d'autant plus sollicitée que fait défaut la connaissance du contexte, et qui, pour sa part de vérité, permet d'insister à nouveau sur une particularité de ce contexte, à savoir la profonde hétérogénéité d'un luthéranisme qui, après 1550 , se distingue surtout par sa grande fragilité. Un des indices de cette fragilité réside dans la difficulté éprouvée face à la diversité des modes d'expression théologique : les luthériens de 1600 semblent ne pas avoir pu parcourir sans un malaise traduit en d'infinies controverses l'espace qui va de l'élaboration systématique des Loci theologici à cette sorte d'Imitation de Jésus-Christ protestante que Arndt leur destinait dans le Von wahrem Christentum. D'autres confessions, à commencer par la catholique, savaient depuis longtemps faire de cette diversité une force. Ce contraste pourrait guider une histoire comparée des catégories de l'édification qui, pour l'époque moderne, rejoint partiellement celle des théologies mystiques.

3 Significativement, la réédition par J.A.S. du premier livre du Von wahrem Christentum paraît dans un méandre de l'édition complète des œuvres de Spener, les études rassemblées de H.S. sur Arndt étant accueillies dans une série de "Travaux sur l'histoire du piétisme ». En l'occurrence, il ne s'agit que d'enveloppes institutionnelles, qui n'orientent pas particulièrement le propos et n'empêchent donc pas de saluer dans ces deux publications une étape dans l'évolution des études arndtiennes. J.A.S., au terme d'une enquête bibliographique exhaustive, a choisi de republier la toute première édition (Francfort sur le Main, 1605) du premier livre du Von wahrem Christentum, et annonce « dans un délai raisonnable » (p. 382) la republication de la première édition intégrale des quatre livres (Magdebourg, 1610), qui quand elle verra le jour comblera une lacune de taille dans notre accès aux documents-clés de la théologie 
du premier XVIIe s. Le choix d'une Urausgabe que Arndt jugeait par ailleurs très fautive (six pages d'émendations sont fournies) pourra alors apparaître dans toute sa pertinence, au regard du permanent travail de reformulation d'un auteur qui s'est toujours montré soucieux d'affirmer sa parfaite conformité à la norme doctrinale de sa confession. À ses critiques, Arndt répondait invariablement par la proclamation d'une fidélité et d'une appartenance. H.S. repart de là : «Arndt als Lutheraner ?»(p. 61-82). La réponse fournie par ses scrupuleuses études, essentiellement biographiques et philologiques (on y appréciera la chronologie détaillée, ainsi que la liste de la « ArndtLiteratur 1700-2005»), est centrée autour de l'affirmation par laquelle on commençait : il se pourrait que Arndt n'ait jamais été un théologien professionnel, au sens d'une formation universitaire régulière (p. 83-134). Sur ce point, la controverse reste ouverte. Comme telle, elle constitue un nouveau symptôme : s'étonner de ce que l'un des principaux théologiens du luthéranisme n'ait pas été docteur, c'est aussi s'engager sur la voie d'une enquête sur les formes de validation professionnelle des appartenances confessionnelles.

Philippe BÜTTGEN (Centre National de la Recherche Scientifique) 